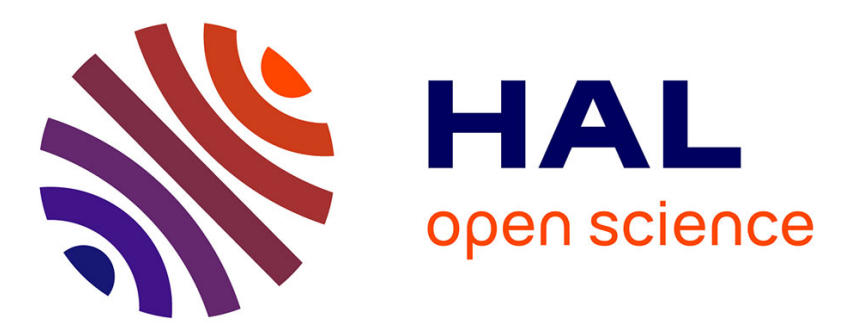

\title{
Energy Efficient Resource Allocation for Underlaying Multi-D2D Enabled Multiple-Antennas Communications
}

\author{
Berna Ozbek, Mylène Pischella, Didier Le Ruyet
}

\section{To cite this version:}

Berna Ozbek, Mylène Pischella, Didier Le Ruyet. Energy Efficient Resource Allocation for Underlaying Multi-D2D Enabled Multiple-Antennas Communications. IEEE Transactions on Vehicular Technology, 2020, 10.1109/TVT.2020.2981409 . hal-02515209

\author{
HAL Id: hal-02515209 \\ https://hal.science/hal-02515209
}

Submitted on 23 Mar 2020

HAL is a multi-disciplinary open access archive for the deposit and dissemination of scientific research documents, whether they are published or not. The documents may come from teaching and research institutions in France or abroad, or from public or private research centers.
L'archive ouverte pluridisciplinaire HAL, est destinée au dépôt et à la diffusion de documents scientifiques de niveau recherche, publiés ou non, émanant des établissements d'enseignement et de recherche français ou étrangers, des laboratoires publics ou privés. 


\title{
Energy Efficient Resource Allocation for Underlaying Multi-D2D Enabled Multiple-Antennas Communications
}

\author{
Berna Özbek, Senior Member, IEEE, Mylene Pischella, Senior Member, IEEE and Didier Le Ruyet, Senior \\ Member, IEEE
}

\begin{abstract}
Energy efficiency has a significant importance to optimize the wireless communications systems by providing high data rates. In order to develop energy efficient systems, one of the promising method is to use multiple device-to-device (D2D) underlaying multiple antenna cellular systems. The interference from cellular users to D2D pairs, the interference between D2D pairs and the interference at the base station (BS) caused by D2D pairs occur in these communications systems. In this paper, we propose energy efficient resource allocation algorithms for underlaying multi-D2D enabled multiple-antennas communications by employing different multiple antenna processing techniques at the BS. A joint method based on Dinkelbach algorithm and Message Passing Algorithm (MPA) and an approach based on deep learning with multi-layer artificial neural network are proposed to maximize the global energy efficiency (GEE) while satisfying the data rate requirements of both cellular users and D2D pairs. In MPA, the factor graph of the D2D pairs is constructed by taking into account the interference among the D2D pairs and the interference level at the BS to avoid any interruption in the cellular transmission. By relying on the training based on the proposed joint algorithm, a deep neural network approach is presented for off-line implementation. The performance results of the proposed energy efficient resource allocation algorithms are illustrated the superiority of multi-D2D communications over conventional single-D2D communications.
\end{abstract}

\section{INTRODUCTION}

Future wireless communication networks will allow users to communicate at much higher data rates to support immersive virtual reality services, cloud-based gaming and real-time HD streaming in addition to conventional multimedia services while designing energy efficient systems [1]. In order to provide these improvements at the radio access level, one solution is to employ device-to-device (D2D) communications underlaying cellular transmission [2] [3]. In the D2D enabled cellular communications systems, this task is challenging due to employing multiple D2D pairs in the same radio resource without interrupting cellular transmission [4] [5]. Therefore, effective resource allocation is required to increase overall system performance considering global energy efficiency (GEE).

Copyright (c) 2015 IEEE. Personal use of this material is permitted. However, permission to use this material for any other purposes must be obtained from the IEEE by sending a request to pubs-permissions@ieee.org.

B. Özbek is with the Department of Electrical-Electronics Engineering, Izmir Institute of Technology, 35430 Izmir, Turkey (e-mail: bernaozbek@iyte.edu.tr).

M. Pischella is with CEDRIC/LAETITIA, CNAM, Paris, France. (e-mail: mylene.pischella@cnam.fr)

D. Le Ruyet is with CEDRIC/LAETITIA, CNAM, Paris, France. (e-mail: leruyet@cnam.fr)
Resource allocation in such a scenario is a combinatorial and highly non-trivial problem. Known near optimal solutions are highly complex, while greedy solutions are suboptimal.

In this paper, we propose a communications system including multi-D2D pairs sharing the same resource block (RB) with a cellular user through the Base Station (BS) with multiple antennas to maximize the GEE under the constraints om maximum transmitted power and required data rate. Since uplink resources are usually underutilized compared to downlink resources in the cellular communications and co-channel interference caused by D2D pairs can be handled more easily by a BS than cellular users, we consider uplink transmission for the underlaying D2D systems.

In order to maximize the achievable energy efficiency, resource allocation and power control has been examined for D2D communications underlaying cellular networks in the literature. In [6], a joint resource allocation and power control has been given to improve the energy efficiency (EE) for each cellular user and D2D pair rather than increase the GEE. In [7] [8] [9] [10], several joint resource allocation (RA) and power control algorithms have been provided to maximize the EE of D2D communications while guaranteeing the quality of service of cellular users. In [11], a channel and power allocation that maximizes the minimum EE of the D2D links has been given. For an energy efficient RA framework with Hybrid Automatic Repeat Request (HARQ), four EE-related optimization problems with a constraint on the minimum goodput and on the maximum transmit power per link have been examined in [12]. In [13] [14], the maximization of EE for D2D communication under the constraints of both D2D pairs and cellular users quality of service has been investigated. The fairness aware energy-efficient RA algorithm has been examined in [15] to improve the EE of individual nodes. However, in all the cited literature, only one D2D pair and one cellular user can be allocated per RB. In the present paper, we perform a resource allocation to maximize GEE by multiplexing multiple D2D pairs to one cellular RB, which causes interference between the D2D pairs as well as cumulative interference at the BS.

In order to maximize sum data rate, the resource allocation algorithms employing multiple D2D pairs for RB sharing with one cellular user have been intensively addressed in the literature. The data rate of D2D pairs has been maximized in [16] through the design of a distributed RA scheme. In [17], a two-phase-based resource sharing algorithm has been 
examined to improve the network throughput. In [18], the interference through power control has been handled for the weigthed sum rate maximization of D2D pairs. The problem of designing a joint uplink subcarrier assignment and power allocation scheme has been investigated in [19] in order to maximize the sum data rate of $\mathrm{D} 2 \mathrm{D}$ pairs while satisfying data rate requirements of cellular users. A joint channel and power allocation in a time-varying environment for underlaying D2D network has been addressed in [20] in a fully distributed approach. In order to maximize overall sum data rate for multi-D2D multiple antennas cellular systems, an interference management algorithm has been presented for the multiuser systems in [21] and Message Passing Algorithm (MPA) [22] based on the resource allocation in [23] has been examined. In contrast to these works, in this paper, we focus on the maximization of the GEE while employing multi-D2D pairs for each $\mathrm{RB}$.

Deep learning with multi-layer Artificial Neural Network (ANN) has raised much attention recently for applications in wireless resource allocation. The recent works in [24] [25] [26] demonstrated that deep learning is one of the promising techniques for resource allocation in fifth generation (5G) and beyond 5G wireless systems. Resource allocation can indeed be seen as a non-linear mapping between some input parameters (that are network and channel-dependent) and the final allocation outcome. For instance, the allocation outputs of the Weighted Minimum Mean Squared Error (WMMSE) algorithm [27] aiming at maximizing the weighted sum rate of a multi-cell interference network have been emulated with an ANN in [28] and with a convolutional neural network in [29]. In [30], the ANN has been trained to perform max-min and max-product power allocation in the downlink of a Massive multiple input multiple output (MIMO) network. In [31], 3 dimensional MPA with its neural network-based deep learning scheme have been recently considered for resource allocation in cognitive radio networks. Some other recent references on ANN for wireless resource allocation can be found in [32].

In the underlaying multi-D2D enabled multiple-antennas cellular communications, three different kind of interferences occur: the interference from cellular users to D2D pairs, the interference between D2D pairs and the interference at the BS caused by D2D pairs. In this work, in order to manage these interferences, we propose energy efficient resource allocation algorithms and our main contributions are summarized as follows:

- The system model including multi-cellular users and multi-D2D pairs is examined in a single cell with a BS with multiple antennas. We derive an optimal GEE formulation under the constraints of transmitted powers and signal-to-interference-noise-ratio (SINR) values for both cellular users and D2D pairs. As this optimization problem is NP-hard, it is divided into sub-problems and solved in a tractable way.

- Cellular users are firstly allocated with the objective to maximize the energy efficiency, as priority is given to cellular transmissions over D2D communications. Since orthogonal RA is assumed for cellular users, only one cellular user is allocated to one RB.
- Once the cellular user is allocated, the GEE optimization problem to allocate multi-D2D pairs for one RB is reformulated. A joint algorithm including a power control based on Dinkelbach algorithm and a resource allocation based on MPA is proposed to solve this problem by employing two different multiple antenna techniques at the BS to mitigate cumulative interference coming from multi-D2D pairs.

- An approach based on Deep learning with multi-layer ANN is also investigated. A training set is generated with the proposed joint Dinkelbach and MPA algorithm. Once trained, the ANN can be used and leads to lowcomplexity online implementation.

The rest of this paper is organized as follows: Section II presents the system model and the optimization problem to maximize GEE. Section III describes the proposed energy efficient resource allocation algorithms. Section IV provides extensive performance results of the proposed algorithms with numerical simulations. Finally, some concluding remarks are drawn in Section V.

\section{System Model And Problem Definition}

We examine uplink underlaying multi-D2D based communications in a single cell including one BS with $N_{r}$ antennas, $K_{c}$ cellular users having single antenna and $K_{d}$ D2D pairs with single antenna as illustrated in Fig 1. While defining system model, we consider only one RB whose bandwidth is $B_{c}$ and allocate only one cellular user and multi-D2D pairs for each RB.

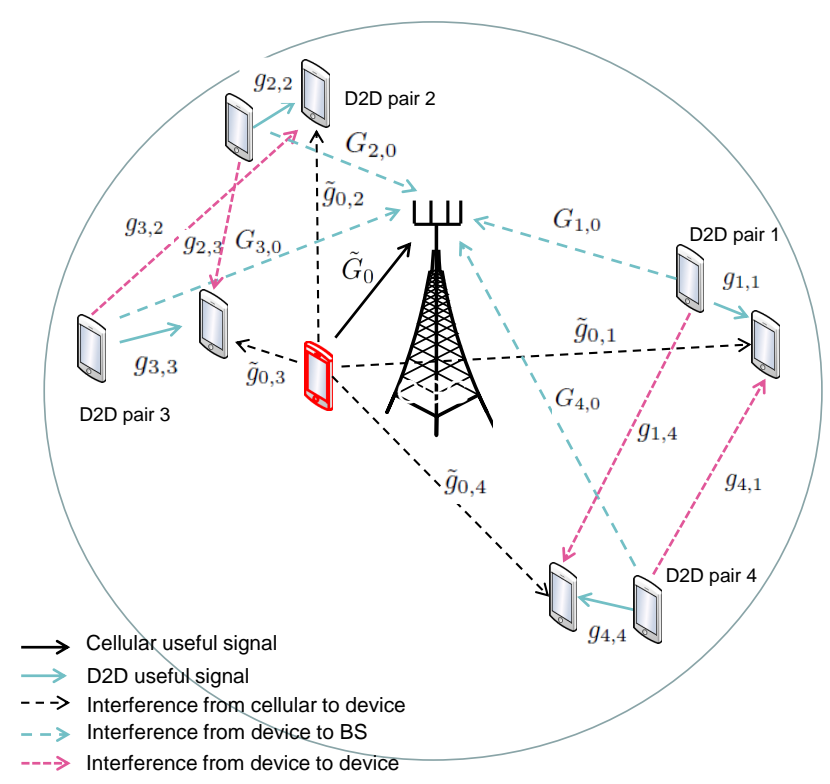

Figure 1: Underlaying multi-D2D enabled cellular communications.

The data rate of $\mathrm{D} 2 \mathrm{D}$ pair $k_{d}$ is determined by,

$$
R_{k_{d}}=\log _{2}\left(1+\gamma_{k_{d}}\right)
$$


where $\gamma_{k_{d}}$ is SINR for D2D pair $k_{d}$ and is calculated by,

$$
\gamma_{k_{d}}=\frac{P_{k_{d}} g_{k_{d}, k_{d}}}{N_{0} B_{c}+J_{k_{d}}+I_{k_{d}}}
$$

In Eq. (2), $P_{k_{d}}$ is the transmitted power of D2D transmitter $k_{d}, g_{a, b}$ is the channel gain from D2D transmitter $a$ to D2D receiver $b$ and $N_{0}$ is the noise power spectral density of the Additive White Gaussian Noise (AWGN) channel.

$I_{k_{d}}$ is the total received interference at the D2D receiver $k_{d}$ generated by all remaining D2D transmitters. This interference is calculated by,

$$
I_{k_{d}}=\sum_{\substack{j=1 \\ j \neq k_{d}}}^{K_{d}} P_{j} g_{j, k_{d}}
$$

$J_{k_{d}}$ is the received interference at the D2D receiver $k_{d}$ generated by cellular transmission. This interference is determined by,

$$
J_{k_{d}}=\sum_{k_{c}=1}^{K_{c}} \tilde{x}_{k_{c}} \tilde{P}_{k_{c}} \tilde{g}_{k_{c}, k_{d}}
$$

where $\tilde{P}_{k_{c}}$ is the transmitted power of cellular user, $\tilde{x}_{k_{c}}$ is the boolean indicator that the cellular user $k_{c}$ is allocated or not and $\tilde{g}_{k_{c}, k_{d}}$ is the channel gain between the cellular user $k_{c}$ and D2D receiver $k_{d}$. In the considered system model, only once cellular user is active, so the summation in Eq. (4) only contains one non-null element.

The data rate of cellular user $k_{c}$ is calculated as,

$$
\tilde{R}_{k_{c}}=\log _{2}\left(1+\tilde{\gamma}_{k_{c}}\right)
$$

where $\tilde{\gamma}_{k_{c}}$ is the SINR of the cellular user $k_{c}$ evaluated after post-coding at the BS and is given by,

$$
\tilde{\gamma}_{k_{c}}=\frac{\tilde{P}_{k_{c}} \tilde{G}_{k_{c}, 0}}{N_{0} B_{c}+I_{k_{c}, 0}}
$$

with $\tilde{G}_{k_{c}, 0}$ the channel gain obtained after applying postcoding at the BS and is given by,

$$
\tilde{G}_{k_{c}, 0}=\left|\tilde{\mathbf{h}}_{k_{c}, 0} \mathbf{w}_{k_{c}, 0}\right|^{2}
$$

where $\tilde{\mathbf{h}}_{k_{c}, 0}$ with $1 \times N_{r}$ as the channel vector between the cellular user $k_{c}$ and the BS and $\mathbf{w}_{k_{c}, 0}$ is the post-coding vector of size $N_{r} \times 1$.

The gain for the multiple antennas system can be achieved by performing Maximum Ratio Combining (MRC) or Minimum Mean Square Error (MMSE) receiver at the BS.

For the MRC case, the post-coding vector is determined only by considering the cellular user $k_{c}$ :

$$
\mathbf{w}_{k_{c}, 0}^{\prime}=\left(\tilde{\mathbf{h}}_{k_{c}, 0}\right)^{H}
$$

For the MMSE receiver, the post-coding vector is obtained by taking into account both the cellular user $k_{c}$ and the D2D pairs to mitigate interference coming from D2D pairs at the BS:

$$
\mathbf{w}_{k_{c}, 0}^{\prime}=\left(\sum_{k_{d}=1}^{K_{d}} x_{k_{d}} P_{k_{d}}\left(\mathbf{h}_{k_{d}, 0}^{H} \mathbf{h}_{k_{d}, 0}\right)+\left(N_{0} B_{c}\right) \mathbf{I}_{N_{r}}\right)^{-1}
$$

where $\mathbf{h}_{k_{d}, 0}$ with $1 \times N_{r}$ as the channel vector between the $\mathrm{D} 2 \mathrm{D}$ transmitter $k_{d}$ and the $\mathrm{BS}, x_{k_{d}}$ is the indicator that the D2D pair $k_{d}$ is active or not.

Then, the post-coding vector is normalized as follows:

$$
\mathbf{w}_{k_{c}, 0}=\frac{\mathbf{w}_{k_{c}, 0}^{\prime}}{\left\|\mathbf{w}_{k_{c}, 0}^{\prime}\right\|}
$$

$I_{k_{c}, 0}$ is the received interference at the BS coming from all D2D transmitters and is calculated as,

$$
I_{k_{c}, 0}=\sum_{k_{d}=1}^{K_{d}} I_{k_{d}, k_{c}, 0}
$$

where $I_{k_{d}, k_{c}, 0}$ is the interference generated by the D2D transmitter $k_{d}$ after postcoding based on the cellular user $k_{c}$ at the BS. This interference is determined as,

$$
I_{k_{d}, k_{c}, 0}=P_{k_{d}} G_{k_{d}, k_{c}, 0}
$$

with $G_{k_{d}, k_{c}, 0}$ the channel gain of D2D pair $k_{d}$ after postcoding at the BS and given by,

$$
G_{k_{d}, k_{c}, 0}=\left|\mathbf{h}_{k_{d}, 0} \mathbf{w}_{k_{c}, 0}\right|^{2}
$$

Let define the set of cellular users and D2D pairs as $\mathcal{S}_{c}=\left\{1, \ldots, K_{c}\right\}$ and $\mathcal{S}_{d}=\left\{1, \ldots, K_{d}\right\}$, respectively. Considering that the optimization variables are the vector of allocation indicators of $\mathbf{X}_{d}=\left[x_{1} \ldots x_{K_{d}}\right], \tilde{\mathbf{X}}_{c}=\left[\tilde{x}_{1} \ldots \tilde{x}_{K_{c}}\right]$ and the vector of power values of $\mathbf{P}_{d}=\left[P_{1} \ldots P_{K_{d}}\right], \tilde{\mathbf{P}}_{c}=$ $\left[\tilde{P}_{1} \ldots \tilde{P}_{K_{c}}\right]$ for the D2D pairs and cellular users respectively, the GEE problem can be defined as follows:

$$
\max _{\mathbf{X}_{d} ; \tilde{\mathbf{X}}_{c} ; \mathbf{P}_{d} ; \tilde{\mathbf{P}}_{c}} \frac{\sum_{k_{c}=1}^{K_{c}} \tilde{x}_{k_{c}} \tilde{R}_{k_{c}}+\sum_{k_{d}=1}^{K_{d}} x_{k_{d}} R_{k_{d}}}{\sum_{k_{c}=1}^{K_{c}} \tilde{x}_{k_{c}}\left(\tilde{P}_{k_{c}}+p^{c}\right)+\sum_{k_{d}=1}^{K_{d}} x_{k_{d}}\left(P_{k_{d}}+p^{d}\right)}
$$

subject to

$$
\begin{aligned}
& \tilde{x}_{k_{c}} \in\{0,1\} \quad \forall k_{c} \in \mathcal{S}_{c} \\
& \sum_{k_{c}=1}^{K_{c}} \tilde{x}_{k_{c}}=1 \\
& \tilde{\gamma}_{k_{c}} \geq \tilde{x}_{k_{c}} \tilde{\gamma}_{\text {th }} \quad \forall k_{c} \in \mathcal{S}_{c} \\
& 0 \leq \tilde{P}_{k_{c}} \leq P_{\max } \quad \forall k_{c} \in \mathcal{S}_{c} \\
& x_{k_{d}} \in\{0,1\} \quad \forall k_{d} \in \mathcal{S}_{d} \\
& \gamma_{k_{d}} \geq x_{k_{d}} \gamma_{\text {th }} \quad \forall k_{d} \in \mathcal{S}_{d} \\
& 0 \leq P_{k_{d}} \leq P_{\max } \quad \forall k_{d} \in \mathcal{S}_{d} \\
& I_{k_{d}, k_{c}, 0} \leq N_{r} I_{\max } \quad \forall k_{c} \in \mathcal{S}_{c}, k_{d} \in \mathcal{S}_{d}
\end{aligned}
$$

where the constraint $(\mathrm{C} 2)$ indicates that only one cellular user is allocated, $\tilde{\gamma}_{\text {th }}$ and $\gamma_{\text {th }}$ are the target SINRs for the cellular user and the D2D pair, respectively, $p^{c}$ and $p^{d}$ are the fixed circuit power for the cellular and D2D transmitters, respectively when the node is active, $P_{\max }$ is the maximum transmitted power at each RB for any node.

$H$ The constraint (C8) represents the interference constraint at the BS for each D2D pair and is proportional to $N_{r}$ and $I_{\max }$ which is the maximum allowed interference level. 
In order to satisfy the SINR requirements defined in the constraints (C3) and (C6), the transmitted power of cellular user $k_{c}$ and D2D pair $k_{d}$ are determined by Eq. (15) and Eq.(16), respectively:

$$
\begin{gathered}
\tilde{P}_{k_{c}}=\min \left\{P_{\max }, \frac{\tilde{\gamma}_{\text {th }} \times\left(N_{0} B_{c}+I_{k_{c}, 0}\right)}{\tilde{G}_{k_{c}, 0}}\right\} \\
P_{k_{d}}=\min \left\{P_{\max }, \frac{\gamma_{\mathrm{th}} \times\left(N_{0} B_{c}+I_{k_{d}}+J_{k_{d}}\right)}{g_{k_{d}, k_{d}}}\right\}
\end{gathered}
$$

\section{Proposed Energy EfFicient Resource AlLOCATION}

In this paper, our objective is to maximize the GEE by considering both cellular and D2D transmissions under the condition that cellular users are allocated prior to the $\mathrm{D} 2 \mathrm{D}$ pairs and under the maximum interference constraint at the BS for each D2D pair. In order to manage the objective, firstly one cellular user is allocated and then multiple D2D pairs are multiplexed in order to maximize the GEE.

\section{A. Resource Allocation for Cellular Users}

In order to maximize the energy efficiency in cellular transmissions while satisfying the constraints (C1) - (C3), we allocate the cellular user having the highest channel gain among $K_{c}$ cellular users. Then, the transmitted power of the selected cellular user is determined to satisfy the constraint (C4). The RA algorithm for cellular transmission is provided in Algorithm 1.

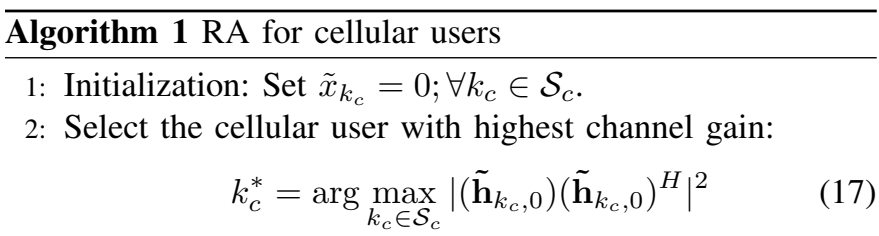

3: Set the allocation indicator of the selected user:

$$
x_{k_{c}^{*}}=1 \text {. }
$$

4: Determine the post-coding vector at the BS using MRC in Eq. (8) considering the selected cellular user $k_{c}^{*}$ since the multiplexed D2D pairs are not known at that stage.

5: Calculate the transmitted power $\tilde{P}_{k_{c}^{*}}$ for the selected cellular user using Eq.(15) by replacing $I_{k_{c}^{*}, 0}$ by $\widehat{I_{0}}$ which is the estimated interference level at the BS and is calculated considering the worst case as:

$$
\widehat{I_{0}}=K N_{r} I_{\max }
$$

6: Calculate the data rate $\tilde{R}_{k_{c}^{*}}$ for the selected cellular user $k_{c}^{*}$ using Eq.(5) with $\tilde{P}_{k_{c}^{*}}$ and $\widehat{I_{0}}$.

In the Step 5 of Algorithm 1, we consider the worst case that all D2D pairs are allocated. This assumption avoids to underestimate the received interference at the $\mathrm{BS}$ since the maximum interference level at the BS is considered.

For the sake of simplification, in the rest of the paper, once the selected cellular user $k_{c}^{*}$ is determined, we will replace
$\tilde{P}_{k_{c}^{*}}$ by $\tilde{P}_{0}, \tilde{R}_{k_{c}^{*}}$ by $\tilde{R}_{0}, \mathbf{w}_{k_{c}^{*}, 0}$ by $\mathbf{w}_{0}, I_{k_{d}, k_{c}^{*}, 0}$ by $I_{k_{d}, 0}, I_{k_{c}^{*}, 0}$ by $I_{0}$ and $G_{k_{d}, k_{c}^{*}, 0}$ by $G_{k_{d}, 0}$.

\section{B. Joint Dinkelbach Method and MPA based RA}

Once the cellular user was allocated to maximize the energy efficiency as described in Algorithm 1, the optimization Problem (14) can be re-written in the following:

$$
\max _{\mathbf{X}_{d} ; \mathbf{P}_{d}} \frac{\tilde{R}_{0}+\sum_{k_{d}=1}^{K_{d}} x_{k_{d}} R_{k_{d}}}{\left(\tilde{P}_{0}+p^{c}\right)+\sum_{k_{d}=1}^{K_{d}} x_{k_{d}}\left(P_{k_{d}}+p^{d}\right)}
$$

subject to

$$
\begin{aligned}
& x_{k_{d}} \in\{0,1\} \quad \forall k_{d} \\
& \gamma_{k_{d}} \geq x_{k_{d}} \gamma_{\mathrm{th}} \quad \forall k_{d} \\
& 0 \leq P_{k_{d}} \leq P_{\max } \quad \forall k_{d} \\
& I_{k_{d}, 0} \leq N_{r} I_{\max } \quad \forall k_{d}
\end{aligned}
$$

In order to tackle Problem (20), one of the widely used approaches is fractional programming, particularly, the popular Dinkelbach's algorithm.

Considering Problem (20) with the set of feasible power values, an auxiliary function $F: \lambda \in \mathbb{R} \rightarrow F(\lambda)$ is defined as:

$$
\begin{aligned}
F(\lambda) & =\left(\tilde{R}_{0}+\sum_{k_{d}=1}^{K_{d}} x_{k_{d}} R_{k_{d}}\right) \\
& -\lambda\left(\left(\tilde{P}_{0}+p^{c}\right)+\sum_{k_{d}=1}^{K_{d}} x_{k_{d}}\left(P_{k_{d}}+p^{d}\right)\right)
\end{aligned}
$$

Dinkelbach algorithm provides the global solution of Problem (20) by iteratively solving Problem (21) and then updating parameter $\lambda$, as described in Algorithm 2.

Problem (21) with the constraints (C5) - (C8) can be solved, at each iteration, with a MPA referred to as Algorithm 3. It will be described in detail in the next section.

\section{Message-Passing Algorithm}

At each iteration $j$, Algorithm 2 requires to solve the following optimization problem:

$$
\max _{\mathbf{X}_{d} ; \mathbf{P}_{d}} F\left(\lambda^{j}\right)
$$

subject to the constraints (C5) - (C8).

The constraints (C6) and (C7) provide the minimum value of $\mathbf{P}_{d}$ for given $\mathbf{X}_{d}$. If $x_{k_{d}}=1$, the power is calculated by Eq. (16). Otherwise, it is equal to 0. Consequently, Problem (24) can be written as a discrete problem as follows:

$$
\min _{\mathbf{X}_{d}}-F\left(\lambda^{j}\right)
$$

subject to the constraints (C5) and (C8).

The discrete optimization Problem (25) can be solved with the min-sum message-passing algorithm, which is a variant of the sum-product message-passing algorithm introduced in [22]. The sum-product algorithm is well-adapted to solve discrete optimization problems with variables where the objective 


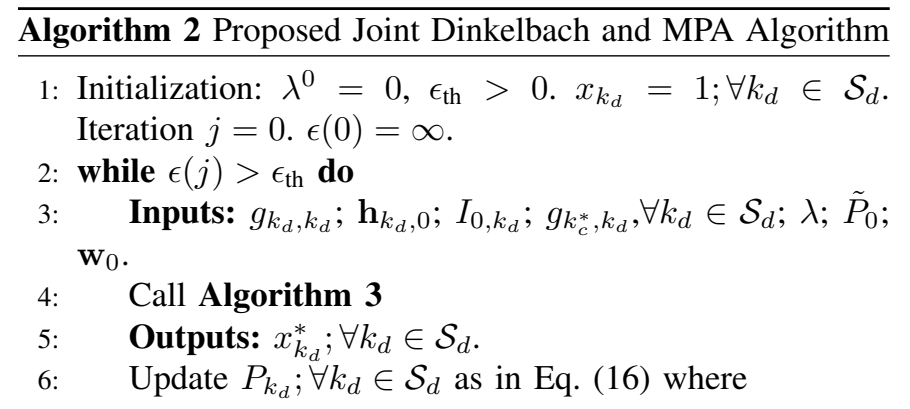

$$
I_{k_{d}}=\sum_{\substack{j=1 \\ j \neq k_{d}}}^{K_{d}} x_{j}^{*} P_{j} g_{j, k_{d}}
$$

7: $\quad$ Update $R_{k_{d}} ; \forall k_{d} \in \mathcal{S}_{d}$ using Eq.(1).

8: When the MMSE receiver is selected to be implemented at the BS, then the post-coding vector is given Eq. (9) is used by considering $x_{k_{d}}^{*} ; \forall k_{d}$.

9: $\quad$ Update $\tilde{R}_{0}$ using Eq.(5) where

$$
I_{0}=\sum_{k_{d}=1}^{K_{d}} x_{k_{d}}^{*} I_{k_{d}, 0}
$$

10: $\quad$ Set the parameter $\lambda$ for the next iteration:

$$
\lambda^{j+1}=\frac{\tilde{R}_{0}+\sum_{k_{d}=1}^{K_{d}} x_{k_{d}}^{*} R_{k_{d}}}{\left(\tilde{P}_{0}+p^{c}\right)+\sum_{k_{d}=1}^{K_{d}} x_{k_{d}}^{*}\left(P_{k_{d}}+p^{d}\right)}
$$

11: Update the optimization function:

$$
\begin{aligned}
F\left(\lambda^{j+1}\right) & =\left(\tilde{R}_{0}+\sum_{k_{d}=1}^{K_{d}} x_{k_{d}}^{*} R_{k_{d}}\right) \\
& -\lambda^{j+1}\left(\left(\tilde{P}_{0}+p^{c}\right)+\sum_{k_{d}=1}^{K_{d}} x_{k_{d}}^{*}\left(P_{k_{d}}+p^{d}\right)\right)
\end{aligned}
$$

$$
\begin{aligned}
& \text { 12: } \quad \epsilon(j+1)=F\left(\lambda^{j+1}\right)-F\left(\lambda^{j}\right) . \\
& \text { 13: } \quad j=j+1 \\
& \text { 14: end while }
\end{aligned}
$$

function can be factorized into a product of several local functions and each function only depends on a subset of the variables. Then the corresponding factor-graph is a by-partite graph that consists on the one hand on variable nodes that represent all the discrete variables of the optimization problem, and on the other hand on factor nodes, that represent the local functions after factorization. A given factor node is connected to a specific variable node by an edge if the local function that it represents depends on this variable. The sum-product algorithm computes the marginal local functions iteratively by exchanging messages between factor nodes and variables nodes. It finally provides the A Posteriori Probability (APP) of the variables. The sum-product algorithm may be replaced by a variant called max-product algorithm, that does not compute all the APP, but only provides the set of variables with the largest APP, thus leading to maximum-likelihood (ML) detection if all variables have the same a priori probability. Finally, the max-product algorithm can be turned into the minsum algorithm by computing the opposite of the logarithm of the objective function.

After having defined the factor-graph for the studied problem, we will show how it can be solved with the min-sum algorithm, and recall of the different messages that must be exchanged between factor nodes and variables nodes.

For the Problem (25), the factor graph is composed of $K_{d}$ variables nodes (one per D2D transmitter) and $K_{d}+1$ factor nodes (one per D2D receiver and the $\mathrm{BS}$ ). Then, the objective function in Problem (25) is a summation of $K_{d}+1$ terms that each depends on a subset of the optimization variables. Given that these subsets contain a low number of elements, Problem (25) can be solved by applying the MPA on a factor graph that represents the interference interactions between all transmitters and receivers.

In order to have a factor graph with low degree, which reduces the complexity of MPA, we neglect some interference while building the factor graph by taking into account given threshold values. Then, the factor graph is constructed as follows: Variable node $a \in \mathcal{S}_{d}$ is always connected to factor node $a$ since it represents active transmission for D2D pair $a$. Factor node $b \in \mathcal{S}_{d}$ is connected to variable node $a \in \mathcal{S}_{d}$, with $a \neq b$, if the estimated interference generated by D2D transmitter $a$ on $\mathrm{D} 2 \mathrm{D}$ receiver $b$ is higher than a given threshold value. Then, the connectivity constraint consequently is given by,

$$
g_{a, b} P_{a}>I_{t h}
$$

where $I_{t h}$ is the threshold on interference and the transmit power of D2D transmitter $k_{d}$ for a given SINR requirement:

$$
P_{k_{d}}=\min \left\{P_{\max }, \frac{\gamma_{\text {th }} \times\left(N_{0} B_{c}+\widehat{I}_{k_{d}}+J_{k_{d}}\right)}{g_{k_{d}, k_{d}}}\right\}
$$

where $\widehat{I}_{k_{d}}$ is the estimated interference level at D2D receiver $k_{d}$. At this stage, the worst case in which all D2D pairs are assumed to be active is considered to avoid any underestimation of interference between D2D pairs. At the D2D receiver $k_{d}$, it is determined as follows:

$$
\widehat{I}_{k_{d}}=K_{d} I_{t h}
$$

Similarly, the BS factor node 0 is connected to variable node $k_{d} \in \mathcal{S}_{d}$ if the estimated interference generated by D2D transmitter $k_{d}$ is larger than a given threshold. This constraint that is proportional to $N_{r}$ and $I_{\max }$, is given by,

$$
G_{k_{d}, k_{c}^{*}, 0} P_{k_{d}}>N_{r} I_{\max }
$$

Since some interference is neglected in underlaying D2D cellular communications system, the factor graph is not fully connected. The complexity of MPA is in $\mathcal{O}\left(2^{c}\right)$, where $c$ is the maximum number of interferers at the $\mathrm{BS}$ or the maximum number of D2D interferers plus one (accounting for the active cellular user) at the $\mathrm{D} 2 \mathrm{D}$ receivers. $c$ will be limited to $c_{\max }$ for complexity reduction purposes. 
Our optimization objective is to determine the optimal values of $x_{k_{d}} \in\{0,1\}$ for each D2D pair, and subsequently the optimal transmit power values of $P_{k_{d}}$ in order to maximize the GEE, given that the cellular user $k_{c}^{*}$ was already allocated. Besides, the maximum interference threshold per D2D pair at the BS must be hold to avoid degradation on the cellular user transmission.

Firstly, we define the variables in the factor graph. The factor and variable nodes are labeled respectively as $M_{i} ; i=$ $0,1, \ldots, K_{d}$ and $x_{j} ; j=1,2, \ldots, K_{d}$ in the factor graph. Since the data rate of D2D receiver $k_{d}$ depends on the vector $\mathbf{X}_{d}$ and some interference is neglected as previously explained, $R_{k_{d}}$ can be expressed as a function of a subset of D2D allocation indicators. Similarly, the data rate at the BS is a function of the subset $\mathcal{X}_{0}$, where this subset is restricted to the variable nodes that are connected to the factor node 0 . Let $\mathcal{X}_{k_{d}}$ be the subset that only contains the allocation indicators of the variable nodes that are connected to the factor node $M_{k_{d}}$ and let $\mathbf{X}_{\mathcal{X}_{i}}$ be the vector obtained by taking only the elements of $\mathbf{X}_{d}$ with the indices in the set $\mathcal{X}_{i}$. Finally, we also define $\mathcal{M}_{k_{d}}$ as the subset that only contains the allocation indicators of the function nodes that are connected to the variable node $x_{k_{d}}$.

For the BS, we can define the optimization function by,

$$
F_{0}\left(\mathcal{X}_{0}\right)=\tilde{R}_{0}\left(\mathcal{X}_{0}\right)-\lambda^{j}\left(\tilde{P}_{0}\left(\mathcal{X}_{0}\right)+p^{c}\right)
$$

If the interference constraint given in (C8) is not fulfilled by D2D transmitter $k_{d}$, then, this D2D transmitter should not be active. Therefore, this condition is included to the optimization objective as a penalty function. For the case of $I_{k_{d}, 0} \leq N_{r} I_{\max }$, the optimization function for D2D pair $k_{d}$ is defined as:

$$
F_{k_{d}}\left(\mathcal{X}_{k_{d}}\right)=R_{k_{d}}\left(\mathcal{X}_{k_{d}}\right)-\lambda^{j}\left(P_{k_{d}}\left(\mathcal{X}_{k_{d}}\right)+p^{d}\right)
$$

Otherwise, this optimization function is determined as follows:

$$
F_{k_{d}}\left(\mathcal{X}_{k_{d}}\right)=-\infty
$$

Then, neglecting some interference terms, the above the optimization Problem (25) with the constraints (C5) and (C8) can be re-written as follows:

$$
\min _{\mathbf{X}_{d}}-\left(F_{0}\left(\mathcal{X}_{0}\right)+\sum_{k_{d}=1}^{K_{d}} F_{k_{d}}\left(\mathcal{X}_{k_{d}}\right)\right)
$$

In order to better illustrate how the factor graph is built, Fig. 2 represents the factor graph corresponding to the scenario depicted on Fig. 1. The variable nodes are pictured by 4 circles, each corresponding to the optimization variable at each of the 4 D2D transmitters, and the factor nodes are represented by 5 squares, the first one (indexed $M_{0}$ ) representing the function to be optimized at the BS, and the four next representing the function to be optimized at each of the $4 \mathrm{D} 2 \mathrm{D}$ receivers. These functions are expressed in Eq. (30) and (31), respectively. In Fig. 2, we assume that some of the interference terms have been neglected and consequently, that the data rate at the BS only depends on the interference received from D2D transmitter 2, but not on the interference received from the other D2D transmitters. This expresses that Eq. (29) only holds for D2D transmitter $k_{d}=2$. Of course, the cellular data rate

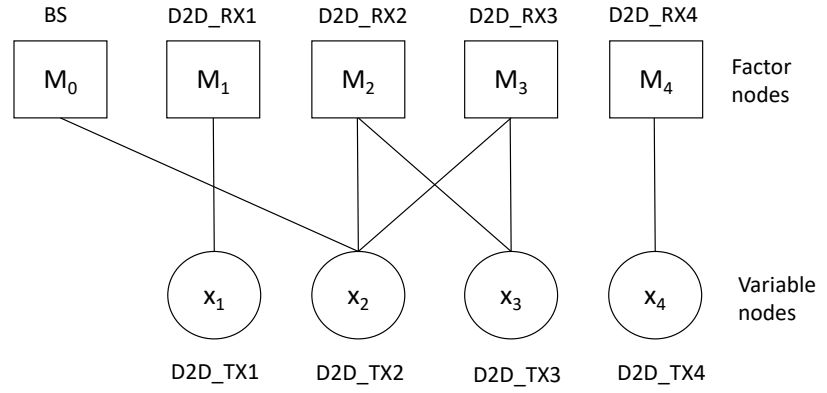

Figure 2: Example of Factor Graph corresponding to the system model in Fig. 1.

at the BS depends on the cellular transmitted power set by Eq. (15), but it is not an optimization variable and therefore does not appear in the factor graph. Finally, even though D2D pairs 4 and 1 are not too far away on Fig. 1, we assume that the interference generated by D2D transmitter 4 on D2D receiver 1 and vice-versa does not fulfill the constraint in Eq. (26). Consequently, variable node $x_{1}$ and factor node $M_{4}$ are not connected in the factor graph, contrary to variable node $x_{2}$ and factor node $M_{3}$. The maximum degree of this factor graph is 2 and even-though the factor graph is not cycle-free, it can be called sparse, as the number of connections is equal to 7 out a possible maximum of 20 connections.

During the execution of MPA, two types of messages are used: messages passed from variable nodes to factor nodes and messages passed from factor nodes to variable nodes. These messages are iteratively exchanged between factor nodes and variable nodes which are connected in the factor-graph for multi-D2D based multiple antenna cellular communications system. At iteration $n$, we denote the message passed from factor node $M_{j}$ to variable node $x_{k_{d}}$ as $\mu_{M_{j} \rightarrow x_{k_{d}}}^{n}\left(x_{k_{d}}\right)$ and denote the message passed from variable node $x_{k_{d}}$ to factor node $M_{j}$ as $\mu_{x_{k_{d}} \rightarrow M_{j}}^{n}\left(x_{k_{d}}\right)$. Besides, $\mathcal{X}_{i} \backslash\{j\}$ represents the set of indices in $\mathcal{X}_{i}$ except index $j$.

Algorithm 3 summarizes the min-sum MPA as proposed by [22] in order to obtain the set of variable following ML optimization.

We can notice that, since the factor graph may contain cycles, the MPA does not necessarily lead to the global optimum of Problem (25) subject to the constraints (C5) and (C8). However, as shown in the simulation results and in other papers using MPA for resource allocation [33] [34] [35] [36], it still achieves performances that are very close to those of exhaustive search.

\section{ANN based RA}

Even though the computational complexity of the proposed joint Dinkelbach and MPA algorithm is not prohibitive, it may be interesting in practical networks not to use it online 


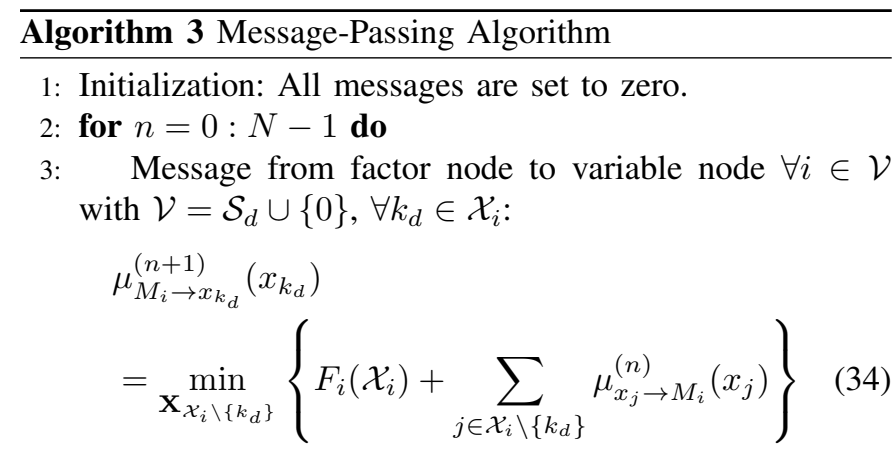

4: $\quad$ Message from variable node to factor node: $\forall k_{d} \in \mathcal{S}_{d}$ and $\forall i \in \mathcal{M}_{k_{d}}$ :

$$
\mu_{x_{k_{d}} \rightarrow M_{i}}^{(n+1)}\left(x_{k_{d}}\right)=\sum_{j \in \mathcal{M}_{k_{d}} \backslash\{i\}} \mu_{M_{j} \rightarrow x_{k_{d}}}^{(n+1)}\left(x_{k_{d}}\right)
$$

5: end for

6: Determine the allocation parameters for all D2D pairs:

$$
x_{k_{d}}^{*}=\arg \min _{x_{k_{d}}}\left\{\sum_{j \in \mathcal{M}_{k_{d}}} \mu_{M_{j} \rightarrow x_{k_{d}}}^{(N)}\left(x_{k_{d}}\right)\right\}
$$

in order to gain some computational time. The behavior of this algorithm can be learned by a deep ANN, by studying the mapping between the system parameters and the obtained allocation values $\mathbf{X}_{d}$ that maximize GEE. Specifically, the resource allocation problem for D2D users in Eq. (20) under the constraints (C5) - (C8) can be modeled as a non-linear map between the system parameters and $\mathbf{X}_{d}$ as follows:

$$
\mathcal{F}_{m}: \mathbf{G} \in \mathbb{R}^{\left(K_{d}+1\right)^{2}} \longmapsto \mathbf{X}_{d}
$$

where the system parameters $\mathbf{G}$ are the channel gains between any $\mathrm{D} 2 \mathrm{D}$ transmitter and any $\mathrm{D} 2 \mathrm{D}$ receiver, the channels gains between any D2D transmitter and the BS, as well as the channels gains between the selected cellular user and any D2D receiver and and the channel gain between the selected cellular user and the BS. In order to improve the learning process, the following pre-processing is performed: all channel gains are divided by the noise power and then expressed in $\mathrm{dB}$. The total number of input parameters consequently is $\left(K_{d}+1\right)^{2}$. We assume that the other parameters $\left(I_{\max }, \gamma_{\mathrm{th}}\right)$ are fixed, but they could of course also be input parameters of the ANN.

If the allocation outcomes are discrete and of reasonable size, they may further be seen as classes. In this paper, we choose the classifying approach [32] in order to optimize the ANN. This approach has not been taken yet in the literature for resource allocation, because most papers used ANN to model power allocation with continuous variables such as [27]-[30]. When seen as a classifying problem, the resource allocation Problem (20) can be modeled as:

$$
\mathcal{F}_{c}: \mathbf{G} \in \mathbb{R}^{\left(K_{d}+1\right)^{2}} \longmapsto C \in \mathcal{C}
$$

where $\mathcal{C}=\left\{C_{0}, C_{1}, \ldots, C_{2^{K}-1}\right\}$ is the set of classes. Each possible vector $\mathbf{X}_{d}$ is equal to a class. The number of classes consequently is $2^{K_{d}}$. Even though this classifying technique may become too complex for large values of $K_{d}$, it is more efficient than directly learning the mapping between the channel gains, that would require larger training sets.

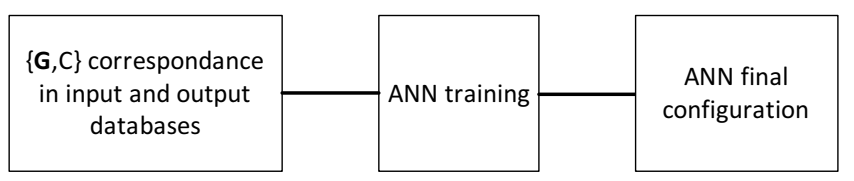

Figure 3: ANN off-line training

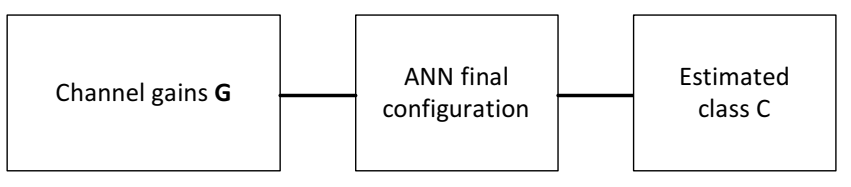

Figure 4: Online resource allocation with ANN.

The chosen ANN is a feed-forward neural network with fully-connected layers. A multi-layer feedforward ANN is generated and optimized. The weights and bias are obtained with the objective to minimize the cross-entropy [37] using the scaled conjugate gradient method [38].

Once the ANN has been trained off-line with a sufficiently large training set, it can be used online in order to emulate the outputs of the proposed joint MPA and Dinkelbach algorithm with low computational complexity, as represented on Fig. 3 and Fig. 4.

\section{Performance Evaluations}

We consider a single cell scenario with cell radius $R=$ $0.5 \mathrm{~km}$. There are $K_{c}=25$ cellular users which are uniformly distributed in the cell In the considered system, only one cellular user is allocated to establish transmission with target SINR of $\tilde{\gamma}_{\text {th }}=20 \mathrm{~dB}$. The target SINR value of D2D pairs is also equal to $\gamma_{\mathrm{th}}=20 \mathrm{~dB}$. D2D transmitters are uniformly located in the cell edge region at a distance from $R / 2$ to $R$ from the BS. For both D2D transmitters and cellular users, the maximum transmit power is fixed to $P_{\max }=11 \mathrm{dBm}$ at one RB. In the proposed joint algorithm in Algorithm 2, the threshold value is set to $\epsilon_{\text {th }}=1.1$. In the MPA described in Algorithm 3, the maximum number of iterations is chosen as $N=3$.

For both cellular users and D2D pairs, the channel is modeled by using Rayleigh fading and a shadowing with lognormal distribution whose parameters depend on whether the receiver is the BS or a device. If the receiver is the BS, the path loss model is $L=128.1+37.6 \log _{10}(d(\mathrm{~km}))$ where $d$ is the distance between the transmitter and receiver and the standard deviation for shadowing is equal to $9 \mathrm{~dB}$. If the receiver is a device, the path loss model is $L=140+36.8 \log _{10}(d(\mathrm{~km}))$ and the standard deviation for shadowing is $4 \mathrm{~dB}$. The power spectrum density of AWGN is $N_{0}=-174 \mathrm{dBm} / \mathrm{Hz}$ and the bandwidth of one RB is $B_{c}=180 \mathrm{kHz}$. The circuit powers $p^{c}$ and $p^{d}$ are set to $100 \mathrm{~mW}$ and $50 \mathrm{~mW}$, respectively. The maximum degree per factor node in the factor graph is $c_{\max }=8$. 
A. Performance Results of Joint Dinkelbach and MPA algorithm

In this section, we provide the performance of the proposed joint algorithm that consists of Algorithm 1 (RA for cellular users) followed by Algorithm 2 (Joint Dinkelbach and MPA algorithm).

Firstly, the effect of threshold values of $I_{\max }$ and $I_{\text {th }}$ on the GEE of the proposed joint algorithm is evaluated.

We analyze the effect of maximum allowed interference per D2D on GEE in Table I by fixing the connectivity constraint as $I_{\text {th }}=-121 \mathrm{dBm}$. While $I_{\max }$ reduces, the GEE is improved at the expense of less number of multiplexed D2Ds. Therefore, we set it as $I_{\max }=-121 \mathrm{dBm}$ which is also equal to $B_{c} N_{0}$.

Table I: The effect of $I_{\max }$ on the GEE.

\begin{tabular}{|c|c|c|c|}
\hline$I_{\max }(\mathrm{dBm})$ & -114 & -121 & -128 \\
\hline GEE & 149.3 & 152.1 & 152.5 \\
\hline \hline Multiplex D2Ds & 3.51 & 3.24 & 2.72 \\
\hline \hline
\end{tabular}

We analyze the effect of the connectivity constraint for $I_{\max }=-121 \mathrm{dBm}$ in Table II. It has an equivalent impact on GEE and the number of multiplexed D2D pairs. In the remaining parts, it is also chosen as $I_{t h}=-121 \mathrm{dBm}$.

Table II: The effect of $I_{\text {th }}$ on the GEE.

\begin{tabular}{|c|c|c|c|}
\hline$I_{\text {th }}(\mathrm{dBm})$ & -114 & -121 & -128 \\
\hline GEE & 151.4 & 152.1 & 152.2 \\
\hline Multiplex D2Ds & 3.65 & 3.24 & 2.99 \\
\hline \hline
\end{tabular}

In Fig. 5, we illustrate cumulative density function (CDF) of GEE of the proposed joint algorithm by employing both MRC and MMSE receiver for underlaying D2D communications with $K_{d}=8$ and $N_{r}=4$. We compare the proposed solution with the one given in [23], that first applies Algorithm 1 and then uses MPA to maximize the sum data rate and with the optimal exhaustive search, where Algorithm 1 is followed by an exhaustive search on resource allocation for D2D aiming at maximizing the GEE. According to the performance results, the GEE of the proposed algorithm is significantly increased compared to the MPA for the sum data rate maximization and gives almost the same GEE results as the exhaustive search.

In Fig. 6, the influence of the number of D2D pairs on the GEE is illustrated for the proposed joint Dinkelbach and MPA algorithm. It is compared with an algorithm that only allocates on D2D pair per RB and performs joint selection of cellular user and D2D pair. The chosen D2D pair and the chosen cellular user are the ones that maximize the GEE. Since the proposed algorithm allocates more than one D2D pairs per $\mathrm{RB}$, it provides much higher GEE performance compared to single-D2D pair allocation. As shown in Table III, the number of multiplexed D2Ds is increased proportional to the number of D2D pairs and the proposed algorithm with MMSE receiver is multiplexed slightly higher number of D2Ds compared to MRC case. As a result, the performance of the proposed joint algorithm is increased with the number of D2D pairs since

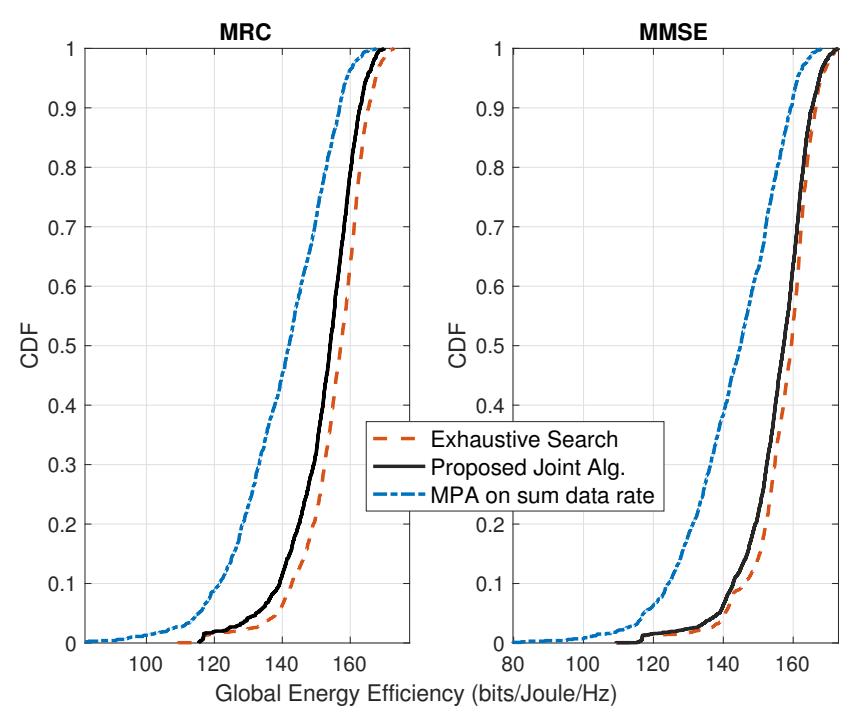

Figure 5: Comparison results of GEE with MRC and MMSE receiver for $N_{r}=4$ and $K_{d}=8$.

the interference levels at D2D receivers as well as at the BS still remain low. In addition to that, we demonstrate that the proposed joint algorithm achieves almost the same GEE performance than the exhaustive search for different number of D2D pairs, while having much less computational complexity thanks to the sparse structure of the factor-graph.

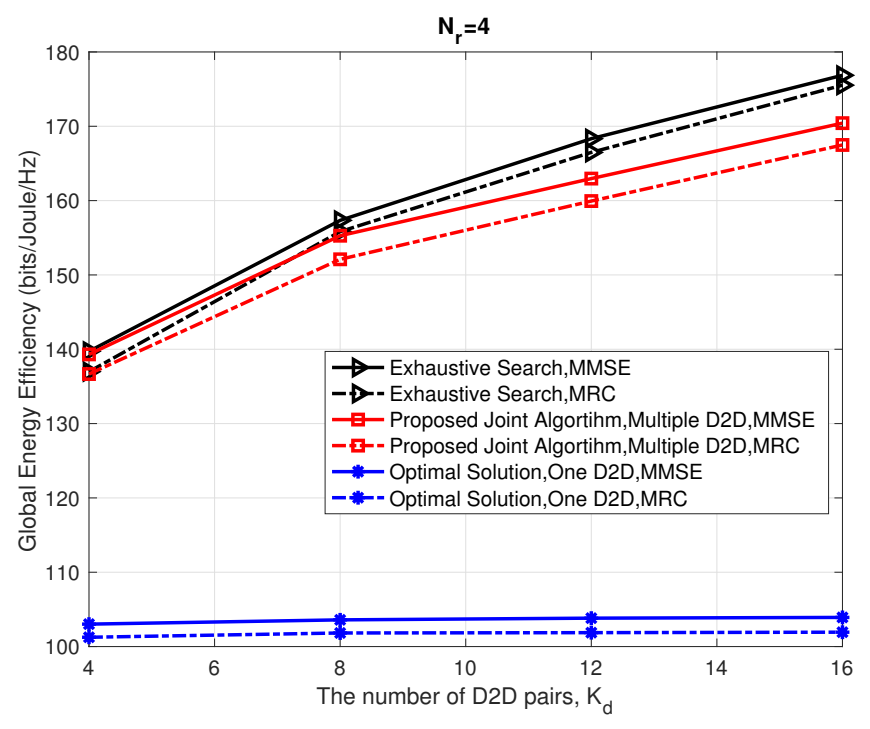

Figure 6: Comparison results of GEE versus different number of D2D pairs for $N_{r}=4$.

Table III: The average number of multiplexed D2D pairs for the proposed joint algorithm with $N_{r}=4$.

\begin{tabular}{|c|c|c|c|c||}
\hline$N_{r}=4$ & $K_{d}=4$ & $K_{d}=8$ & $K_{d}=12$ & $K_{d}=16$ \\
\hline Multiplexed D2Ds,MRC & 2.31 & 3.27 & 3.72 & 3.95 \\
\hline Multiplexed D2Ds,MMSE & 2.34 & 3.32 & 3.83 & 4.09 \\
\hline \hline
\end{tabular}


In Fig. 7, the effect of the number of antennas at the BS on the GEE is illustrated for the proposed joint Dinkelbach and MPA algorithm considering different number of D2D pairs in the underlaying multi-D2D communications. It is shown that GEE is improved by increasing the number of antennas at the BS since the proposed joint algorithm is more robust to the interference coming from D2D pairs. Because, the maximum interference threshold level increases proportionally with the number of antennas at the BS through $N_{r} I_{\max }$. Besides, the proposed joint algorithm with MMSE receiver provides higher GEE when the number of antennas is increased by further mitigating the interference coming from multiplexed D2Ds at the BS. As shown by Fig. 8, compared to the single antenna case, the number of multiplexed D2D increases proportionally with the number of antennas.

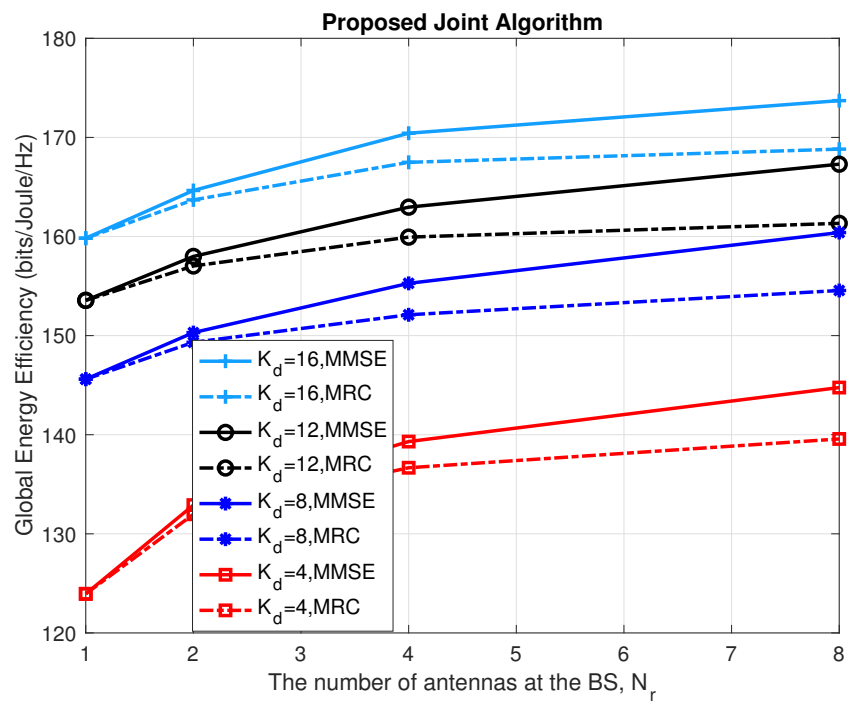

Figure 7: The effect of number of antennas at the BS on the GEE for the proposed joint algorithm.

The complexity of the proposed joint algorithm depends on the number of iterations in Dinkelbach algorithm and on the maximum degree of factor nodes in the factor graph. As given in the Table IV, the complexity of the proposed joint algorithm is very low compared to that of exhaustive search, even though it is able to reach almost the same GEE performance, as seen on Fig. 5 and Fig. 6. Besides, the complexity of Dinkelbach algorithm is further decreased through the proposed joint algorithm with MMSE receiver.

Table IV: The complexity analysis of the proposed joint algorithm with $N_{r}=4$ for different number of D2D pairs.

\begin{tabular}{|c|c|c|c|c|}
\hline & $K_{d}=4$ & $K_{d}=8$ & $K_{d}=12$ & $K_{d}=16$ \\
\hline Max. degree of factor nodes & 2 & 3.2 & 5.5 & 7.5 \\
\hline \hline Iterations in Dinkelbach,MRC & 4.4 & 4.3 & 4.2 & 4.1 \\
\hline Iterations in Dinkelbach,MMSE & 4.2 & 4.1 & 4.15 & 4.05 \\
\hline \hline
\end{tabular}

\section{B. Performance Results for ANN based RA}

The ANN based RA presented in section III-D is tested with $K_{d}=4$ and $K_{d}=8$ with 3 layers and with training

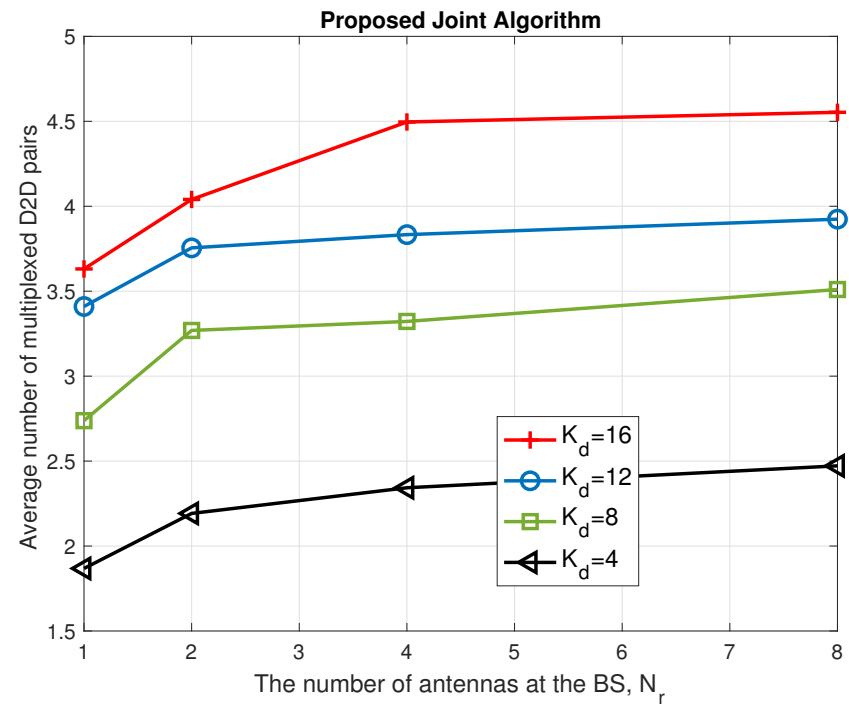

Figure 8: The average number of multiplexed D2D pairs for different number of antennas at the BS for the proposed joint algorithm with MMSE receiver.

sets of 30000 samples obtained through the proposed joint algorithm with MRC receiver. When $K_{d}=4$, the number of classes is 16 . The number of neurons per layer has been arbitrarily set to 20,15 and 10 . Fig. 9 shows the CDF of the GEE on a test set when $K_{d}=4$ (corresponding to 16 classes). Online classifying with ANN almost perfectly emulates the complete joint Dinkelbach and MPA algorithm, leading to a tight match between both CDFs. The difference in terms of average GEE is only $0.11 \%$ compared to using the complete joint Dinkelbach and MPA algorithm. Therefore, ANN-based RA is a very effective strategy for low values of $K_{d}$. Fig. 10 represents the CDF of the GEE when $K_{d}=8$ (corresponding to 64 classes). The number of neurons per layer is equal to 63, 45 and 27. Even though Fig. 10 shows that the CDF of the GEE with online clustering sligthly differs from that obtained with off-line clustering at medium values of the GEE, the difference on the average GEE is still very low, as it is only equal to $1.81 \%$. These results validate the use of classifying with ANN for practical online implementation.

\section{CONCLUSION}

In this paper, we have examined an energy efficient framework for underlaying multi-D2D multi-antenna communications systems. In order to manage the various sources of interferences, we have proposed a joint resource allocation algorithm based on Dinkelbach and MPA approaches to increase GEE by employing MRC and MMSE receiver while satisfying data rate requirements of both cellular user and D2D pairs. In the MPA, the factor graph of the D2D pairs is constructed based on the interference level at the BS and between D2D pairs. The proposed algorithm allocates several D2D pairs on the same radio resource, while guaranteeing the interference constraint at the BS. We have shown that the joint proposed algorithm provides almost the same performance than exhaustive search and outperforms underlaying single 


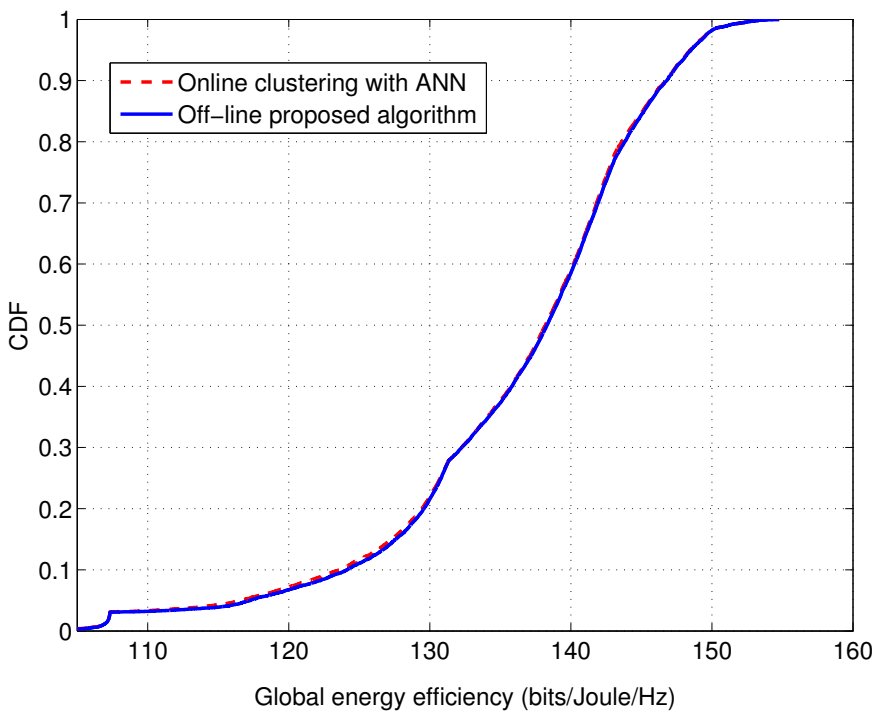

Figure 9: Comparison of off-line and online algorithms when $K_{d}=4$.

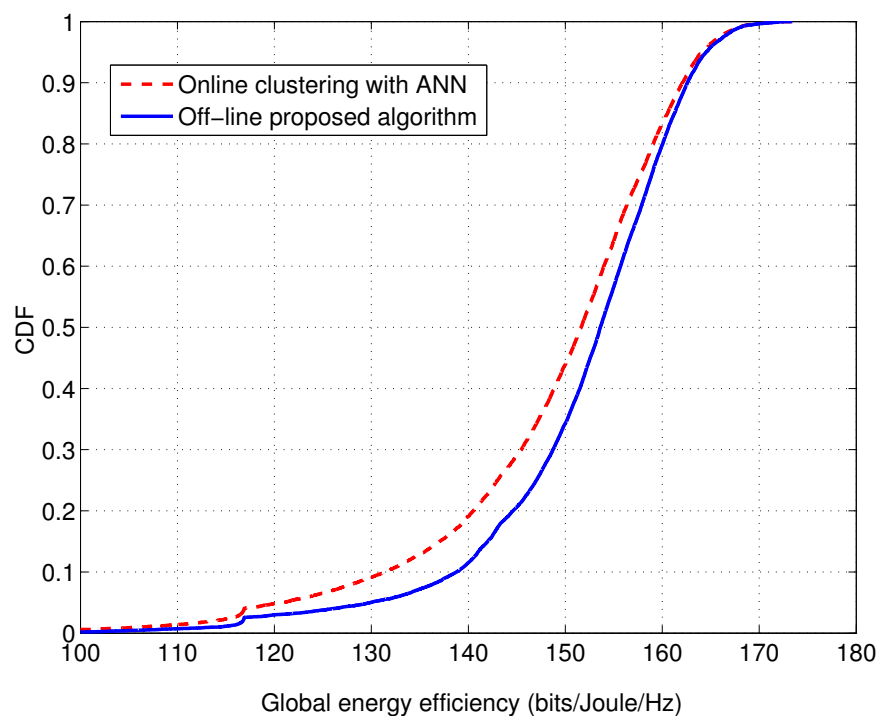

Figure 10: Comparison of off-line and online algorithms when $K_{d}=8$.

D2D multi-antenna cellular systems. Since the convergence time in Dinkelbach algorithm is very short and the constructed factor graph in MPA is not fully connected, the complexity of the proposed algorithm is significantly low compared to the exhaustive search. We have also proposed a deep learning approach with multi-layer ANN based RA that uses offline classifying, and can then be used online for practical implementation. As a future work, we will extend the proposed framework to the case of multiuser multiantenna multicell systems in order to increase GEE of underlaying multi-D2D cellular communications.

\section{REFERENCES}

[1] S. Buzzi, C. I, T. E. Klein, H. V. Poor, C. Yang, and A. Zappone, "A survey of energy-efficient techniques for $5 \mathrm{~g}$ networks and challenges ahead," IEEE Journal on Selected Areas in Communications, vol. 34, no. 4, pp. 697-709, April 2016.

[2] G. Fodor, E. Dahlman, G. Mildh, S. Parkvall, N. Reider, G. Miklós, and Z. Turányi, "Design aspects of network assisted device-to-device communications," IEEE Communications Magazine, vol. 50, no. 3, pp. 170-177, March 2012.

[3] D. Feng, L. Lu, Y. Yuan-Wu, G. Y. Li, G. Feng, and S. Li, "Device-todevice communications underlaying cellular networks," IEEE Transactions on Communications, vol. 61, no. 8, pp. 3541-3551, August 2013.

[4] C. Yu, K. Doppler, C. B. Ribeiro, and O. Tirkkonen, "Resource sharing optimization for device-to-device communication underlaying cellular networks," IEEE Transactions on Wireless Communications, vol. 10, no. 8, pp. 2752-2763, August 2011.

[5] B. Ozbek, M. Pischella, and D. Le Ruyet, "Dynamic shared spectrum allocation for underlaying device-to-device communications," IEEE Wireless Communications, vol. 24, no. 5, pp. 88-93, October 2017.

[6] F. Wang, C. Xu, L. Song, and Z. Han, "Energy-efficient resource allocation for device-to-device underlay communication," IEEE Transaction on Wireless Communications, vol. 14, no. 4, pp. 2082-2092, April 2015.

[7] Y. Jiang, Q. Liu, F. Zheng, X. Gao, and X. You, "Energy-efficient joint resource allocation and power control for $\mathrm{d} 2 \mathrm{~d}$ communications," IEEE Transaction on Vehicular Technology, vol. 65, no. 8, pp. 6119-6127, August 2016.

[8] Z. Zhou, K. Ota, M. Dong, and C. Xu, "Energy-efficient matching for resource allocation in d2d enabled cellular networks," IEEE Transaction on Vehicular Technology, vol. 66, no. 6, pp. 5256-5268, June 2017.

[9] J. Hu, W. Heng, X. Li, and J. Wu, "Energy-efficient resource reuse scheme for $\mathrm{d} 2 \mathrm{~d}$ communications underlaying cellular network," IEEE Communications Letters, vol. 21, pp. 2097-2100, September 2017.

[10] H. Xu, W. Xu, Z. Yang, Y. Pan, J. Shi, and M. Chen, "Energyefficient resource allocation in $\mathrm{d} 2 \mathrm{~d}$ underlaid cellular uplinks," IEEE Communications Letters, vol. 21, no. 3, pp. 560-563, March 2017.

[11] T. D. Hoang, L. B. Le, and T. Le-Ngoc, "Energy-efficient resource allocation for $\mathrm{d} 2 \mathrm{~d}$ communications in cellular networks," IEEE Transaction on Vehicular Technology, vol. 65, no. 9, pp. 6972-6986, September 2016.

[12] X. Leturc, P. Ciblat, and C. J. Le Martret, "Energy-efficient resource allocation for harq with statistical csi," IEEE Transactions on Vehicular Technology, vol. 67, no. 12, pp. 11936-11949, December 2018.

[13] S. Liu, Y. Wu, L. Li, X.I. Liu, and W. Xu, "A two-stage energyefficient approach for joint power control and channel allocation in $\mathrm{d} 2 \mathrm{~d}$ communication," IEEE Access, vol. 7, pp. 16940-16951, January 2019.

[14] R. Zhang, C. Qi, Y. Li, Y. Ruan, C.-X. Wang, and H. Zhang, "Towards energy-efficient underlaid device-to-device communications: A joint resource management approach," IEEE Access, vol. 7, pp. 31385 31396, March 2019.

[15] S. Guo, X. Zhou, S. Xiao, and M. Sun, "Fairness-aware energy-efficient resource allocation in d2d communication networks," IEEE Systems Journal, vol. 13, no. 2, pp. 1273-1284, June 2019.

[16] H. Nguyen, M. Hasegawa, and W. Hwang, "Distributed resource allocation for $\mathrm{d} 2 \mathrm{~d}$ communications underlay cellular networks," IEEE Communications Letters, vol. 20, no. 5, pp. 942-945, May 2016.

[17] H. H. Esmat, M. M. Elmesalawy, and I. I. Ibrahim, "Adaptive resource sharing algorithm for device-to-device communications underlaying cellular networks," IEEE Communications Letters, vol. 20, no. 3, pp. 530-533, March 2016.

[18] M. Pischella, R. Zakaria, and D. le Ruyet, "Resource block level power allocation in asynchronous multi-carrier $\mathrm{d} 2 \mathrm{~d}$ communications," IEEE Communications Letters, vol. 21, no. 4, pp. 813-816, April 2017.

[19] C. Kai, H. Li, L. Xu, Y. Li, and T. Jiang, "Joint subcarrier assignment with power allocation for sum rate maximization of $\mathrm{d} 2 \mathrm{~d}$ communications in wireless cellular networks," IEEE Transactions on Vehicular Technology, vol. 68, no. 5, pp. 4748-4759, May 2019.

[20] S. Dominic and L. Jacob, "Distributed resource allocation for $\mathrm{d} 2 \mathrm{~d}$ communications underlaying cellular networks in time-varying environment," IEEE Communications Letters, vol. 22, no. 2, pp. 388-391, February 2018.

[21] B. Özbek, M. Pischella, and D. Le Ruyet, "Interference management for multiuser multiantenna ofdma underlaying device-to-device communications," in International Symposium on Wireless Communication Systems (ISWCS), Italy, August 2017.

[22] F. Kschischang, B. J. Frey, and H. A. Loeliger, "Factor graphs and the sum-product algorithm," IEEE Transactions on Information Theory, vol. 47, no. 2, pp. 498 - 519, February 2001

[23] B. Özbek, M. Pischella, and D. Le Ruyet, "Message-passing algorithm for sum-rate maximization in multi-antenna underlay $\mathrm{d} 2 \mathrm{~d}$ communica- 
tions," in 2018 International Symposium on Wireless Communication Systems (ISWCS), Portugal, August 2018.

[24] G. Gui, H. Huang, Y. Song, and H. Sari, "Deep learning for an effective nonorthogonal multiple access scheme," IEEE Transactions on Vehicular Technology, vol. 67, no. 9, pp. 8440-8450, September 2018.

[25] M. Liu, T. Song, G. Gui, J. Hu, and H. Sari, "Deep cognitive perspective: resource allocation for noma based heterogeneous iot with imperfect sic," IEEE Internet of Things, vol. 6, no. 2, pp. 2885-2894, April 2019.

[26] G. Gui, H. Huang, Y. Song, and H. Sari, "Data-driven deep learning for automatic modulation recognition in cognitive radios," IEEE Transactions on Vehicular Technology, vol. 68, no. 4, pp. 4074-4077, April 2019.

[27] Q. Shi, M. Razaviyayn, Z. Luo, and C. He, "An iteratively weighted mmse approach to distributed sum-utility maximization for a mimo interfering broadcast channel," IEEE Transactions on Signal Processing, vol. 59, no. 9, pp. 4331-4340, September 2011.

[28] H. Sun, X. Chen, Q. Shi, M. Hong, X. Fu, and N. D. Sidiropoulos, "Learning to optimize: Training deep neural networks for interference management," IEEE Transactions on Signal Processing, vol. 66, no. 20, pp. 5438-5453, October 2018.

[29] W. Lee, M. Kim, and D. Cho, "Deep power control: Transmit power control scheme based on convolutional neural network," IEEE Communications Letters, vol. 22, no. 6, pp. 1276-1279, June 2018.

[30] L. Sanguinetti, A. Zappone, and M. Debbah, "Deep learning power allocation in massive mimo," in 52nd Asilomar Conference on Signals, Systems, and Computers, USA, October 2018.

[31] M. Liu, T. Song, J. Hu, J. Yang, and G. Gui, "Deep learning-inspired message passing algorithm for efficient resource allocation in cognitive radio networks," IEEE Transactions on Vehicular Technology, vol. 68, no. 1, pp. 641-653, January 2019.

[32] Al. Zappone, X. Di Renzo, and M. Debbah, "Wireless networks design in the era of deep learning: Model-based, ai-based, or both?," IEEE Transactions on Communications, vol. 67, no. 10, pp. 7331-7376, October 2019.

[33] I. M. Guerreiro, D. Hui, and C. C. Cavalcante, "A distributed approach to precoder selection using factor graphs for wireless communication networks," EURASIP Journal on Applied Signal Processing, , no. 83, 2013.

[34] A. Abrardo, M. Belleschi, P. Detti, and M. Moretti, "Message passing resource allocation for the uplink of multi-carrier multi-format systems," IEEE Transactions on Wireless Communications, vol. 11, no. 1, pp. 130 141, January 2012.

[35] M. Hasan and E. Hossain, "Distributed resource allocation for relayaided device-to-device communication: A message passing approach," IEEE Transactions on Wireless Communications, vol. 13, no. 11, pp. 6326-6341, November 2014.

[36] Y. Yu, M. Pischella, and D. Le Ruyet, "Distributed antenna selection with message passing algorithm for mimo $\mathrm{d} 2 \mathrm{~d}$ communications," in nternational Symposium on Wireless Communication Systems (ISWCS), Bologna, Italy, September 2017.

[37] J. E. Shore and R. M. Gray, "Minimum cross-entropy pattern classification and cluster analysis," IEEE Transactions on Pattern Analysis and Machine Intelligence, vol. PAMI-4, no. 1, pp. 11-17, January 1982.

[38] M.Fodslette Moller, "A scaled conjugate gradient algorithm for fast supervised learning," Neural Networks, vol. 6, no. 4, pp. 525-533, 1993. 